\title{
Femtosecond Laser-Assisted Big-Bubble Deep Anterior Lamellar Keratoplasty
}

This article was published in the following Dove Press journal:

Clinical Ophthalmology

\author{
Emilio Pedrotti $\mathbb{1}^{\prime}$ \\ Erika Bonacci' \\ Arianna De Rossi ${ }^{1}$ \\ Jacopo Bonetto' \\ Chiara Chierego' \\ Adriano Fasolo ${ }^{1,2}$ \\ Alessandra De Gregorio ${ }^{3}$ \\ Giorgio Marchini' \\ 'Ophthalmic Unit, Department of \\ Neurosciences, Biomedicine and \\ Movement Sciences, University of \\ Verona, Policlinico G.B. Rossi, Verona, \\ 37I34, Italy; ${ }^{2}$ Research Unit, The Veneto \\ Eye Bank Foundation, Venezia, 30174, \\ Italy; ${ }^{3}$ Ophthalmic Unit, San Bassiano \\ Hospital, Bassano Del Grappa, 3606I, \\ Vicenza, Italy
}

Purpose: To determine whether type 1 big-bubble (BB) formation is influenced by the sequence of incisions created with the Victus femtosecond laser (FSL) enabled with software version 3.4 (SV 3.4) during deep anterior lamellar keratoplasty (DALK).

Materials and Methods: Consecutive FSL-assisted DALK BB procedures were performed on 20 human donor corneas: 10 shaped by tunnel incision followed by lamellar incision (tunnel-lamellar group, TL) and 10 in the reverse order (lamellar-tunnel group, LT). The BB type was assessed by evaluating dynamic air movement during air inflation; bubble diameter and floor thickness were measured by anterior segment optical coherence tomography.

Results: Overall, a type $1 \mathrm{BB}$ formed in $85 \%$ of eyes: $100 \%$ in the TL group and $70 \%$ in the LT group. In the LT group, a type $2 \mathrm{BB}$ formed in 2 corneas and one cornea was perforated during cannula insertion. Type 1 BB was achieved after one attempt in $90 \%$ of eyes in the TL group and in $57 \%$ in the LT group.

Conclusion: Shaping the tunnel before rather than after lamellar incision may be more effective for obtaining a type $1 \mathrm{BB}$ by air injection.

Keywords: deep anterior lamellar keratoplasty, femtosecond laser, big bubble, intrastromal incision, lamellar incision

\section{Introduction}

Deep anterior lamellar keratoplasty (DALK) is effective in treating corneal disorders of the anterior corneal layers, such as keratoconus (KC), dystrophy, and scarring. ${ }^{1}$ Compared to penetrating keratoplasty, DALK minimizes the risk of endothelial rejection and assures similar outcomes in visual acuity. ${ }^{2}$ The bigbubble (BB) technique, in which air is injected into the corneal stroma to separate the posterior lamellae, achieves a smooth cleavage plane between the deep stroma layer and the Descemet membrane (DM). ${ }^{3}$

The ideal cleavage plane for BB DALK is determined after identification of the pre-Descemetic layer (PDL). In their study of the dynamics of BB formation by pneumo-dissection, Dua and coworkers discriminated between a type 1 smalldiameter (maximum $8.5 \mathrm{~mm}$ ) bubble between the deep stroma and the PDL, a type 2 large-diameter bubble (maximum $10.5 \mathrm{~mm}$ ) separating the PDL from the DM, and a type 3 bubble in which types 1 and 2 form simultaneously. The type of bubble can be further differentiated by the pattern of air spreading from the center to the periphery and from the periphery to center in type 1 and type $2 \mathrm{BB}$, respectively. ${ }^{4}$

Because the weakened wall is composed of only DM and endothelium, a type 2 $\mathrm{BB}$ is associated with a greater risk of perforation during surgery, formation of
The Veneto Eye Bank Foundation, Pad. G. Rama, via Paccagnella II, Venice, 30174, Italy

Tel +390419656400

Fax+39041965640I

Email adriano.fasolo@yahoo.it 
a double anterior chamber in the postoperative period, ${ }^{5}$ and poor stability of the anterior chamber after a triple procedure. ${ }^{6}$ Differently, exposure of a clear, regular cleavage plane in type $1 \mathrm{BB}$ strengthens the recipient eye, with less risk of complications. ${ }^{7,8}$

The overall success rate of $\mathrm{BB}$ formation depends primarily on the underlying conditions of the recipient cornea and the surgeon's expertise. In patients with $\mathrm{KC}$, $\mathrm{BB}$ formation after manual dissection is reported in about $73 \%$ to $95 \%$ of cases, and type 1, type 2, and type 3 bubble is achieved in about $88 \%, 10 \%$, and $2 \%$ of cases, respectively. ${ }^{9-11}$ Advanced keratoconus was found to be predictive of type $2 \mathrm{BB} ;{ }^{9} \mathrm{BB}$ formation is reported to be $64 \%$ in eyes with corneal scars. ${ }^{12}$

Femtosecond laser (FSL) technology for full- and partial-thickness corneal cuts of different depths and diameters provides highly consist results in a variety of corneal surgeries. ${ }^{13}$ In the context of DALK, it allows for the creation of planar lamellar and side cut of different diameter and predefined corneal depth and location, with ease of dissection of the diseased cornea from the remaining stromal bed. Following FSL-assisted DALK, favorable visual and refractive outcomes have been reported in patients with keratoconus and corneal ectasia, as FSL incisions (eg, mushroom-shaped incision) provide for precise donor-recipient matching, better wound healing, and lower astigmatism. ${ }^{14,15}$

In eyes with keratoconus, the use of FSL software for keratoplasty achieved type $1 \mathrm{BB}$ in $90 \%$ and $\mathrm{BB}$ of both types 1 and 2 in 94\% of patients undergoing DALK. ${ }^{16,17}$ A pilot study using new FSL software technology approved for intracorneal ring segment implantation reported that type $1 \mathrm{BB}$ was achieved in $100 \%$ of cases. ${ }^{18}$ For this study, we evaluated ex vivo a new software program for sequence lamellar and tube-like (named channel or tunnel) incision specifically developed for FSLassisted DALK. The aim was to determine whether the order of creating the two cuts influences the type of BB and its overall formation rate.

\section{Materials and Methods}

For this study, we obtained human donor corneas unsuitable for transplantation from the Veneto Eye Bank Foundation (FBOV, Venice, IT) following approval by the Institutional Review Board of the FBOV (protocol no. CRT/19 rev.02, 24 May 2018) and signed consent from the donor's next of kin for the tissues to be used for research purposes. The study adhered to the tenets of the Declaration of Helsinki.

The corneas were maintained at room temperature in a dextran-containing medium; they were carefully washed with balanced salt solution (BSS) before mounting on a holder (artificial anterior chamber, Network Medical, UK), endothelium facing down. A BSS bottle was connected to the cornea holder and set to a height of $50 \mathrm{~cm}$ to maintain an intracameral pressure of 10 to $20 \mathrm{mmHg}$.

The corneas were cut using an $80 \mathrm{kHz}$ Victus FSL (Technolas Perfect Vision, DE) equipped with software version 3.4 (SV-3.4), a program developed for intracorneal incision including DALK and integrated with imageguided procedure planning and intraoperative monitoring by intraoperative optical coherence tomography (iOCT). The SV-3.4 allows for creating sequential corneal incisions without the need for repeated docking. Lamellar incision provides vertical and horizontal cuts of the stromal layers, while tunnel incision creates a guide for the insertion of a cannula into the deep stroma for pneumo-dissection. For safety reasons, the cutting depth is programmed to a minimum distance of $125 \mu \mathrm{m}$ from the posterior side of the cornea. While performing the BB technique in this study, we disabled the bed cut to get closer to the endothelium. The parameters for each incision were entered following eye docking, and the expected results were displayed in real-time iOCT before starting the cut.

The corneas were randomly allocated for shaping the tunnel incision first and then the lamellar incision (TL group) or in reverse order (LT group). The iOCT images of each step in the procedure were recorded. Following BB formation, the cornea was everted and the floor thickness of the bubble was measured by AS-OCT (Visante, Carl Zeiss Meditec, DE).

\section{Tunnel Incision}

A tunnel was cut in the paracentral position at $130^{\circ}$ to aid cannula insertion; it was composed of two planes: plane 1 angulated with respect to the corneal surface and plane 2 parallel to the corneal surface. The depth of plane 2 was adjusted to end $100 \mu \mathrm{m}$ above the endothelium to minimize the risk of perforation (Table 1).

\section{Lamellar Incision}

A mushroom configuration for cutting the corneas was applied because it has proven reliable when performing DALK. ${ }^{19}$ The mushroom incision was customized to each donor cornea and the cutting depth of the posterior 
Table I Femtosecond Laser Parameters for Tunnel Incision

\begin{tabular}{|l|l|l|}
\hline Parameter & Value & Description \\
\hline Position & $130^{\circ}$ & Axial position of the incision \\
\hline Angle of plane I & $35^{\circ}$ & Angle at the entry of the incision \\
\hline Length of plane 2 & $0.4 \mathrm{~mm}$ & $\begin{array}{l}\text { Length of the posterior parallel part } \\
\text { of the incision }\end{array}$ \\
\hline Outer width & $0.5 \mathrm{~mm}$ & Width at the entry of the incision \\
\hline Inner width & $0.4 \mathrm{~mm}$ & $\begin{array}{l}\text { Final width at the endpoint of the } \\
\text { incision }\end{array}$ \\
\hline
\end{tabular}

Table 2 Femtosecond Laser Parameters for Mushroom Lamellar Incision

\begin{tabular}{|l|l|l|}
\hline Parameter & Value & Description \\
\hline Anterior diameter & $9.0 \mathrm{~mm}$ & $\begin{array}{l}\text { Diameter of the MLKP at the } \\
\text { anterior surface of the cornea }\end{array}$ \\
\hline $\begin{array}{l}\text { Posterior } \\
\text { diameter }\end{array}$ & $6.0 \mathrm{~mm}$ & $\begin{array}{l}\text { Diameter of the corneal bed } \\
\text { resulting from pachymetry, diameter } \\
\text { and side cut angle }\end{array}$ \\
\hline Depth ratio & $70 \%$ & Ratio of cutting depth to pachymetry \\
\hline
\end{tabular}

diameter was set at $70 \%$ of the thinnest point as measured by iOCT. The cutting depth was calculated from the pachymetry measure and the depth ratio (Table 2).

\section{Pneumo-Dissection}

Following FSL incision, the cornea was viewed under a surgical microscope (OPMI Lumera 700, Carl Zeiss, DE) and the anterior stromal lamella was removed. Fogla's cannula was gently inserted bevel down into the end of the tunnel plane 1 incision and the entire length of plane 2. When the inner end of the tunnel was reached, the tip of the cannula was carefully advanced to find the plane of minimum resistance and delaminate it. Air was then gently introduced with a $5-\mathrm{mL}$ syringe to create a BB. A single surgeon (E.P.) performed all dissections.

\section{Evaluation of the Bubble}

The formation and the type of a BB were evaluated intraoperatively according to the air diffusion pattern and the bubble width. At the end of the procedure, the BB was examined by cornea eversion and the thickness of the bubble floor was measured by AS-OCT.

\section{Statistics}

Descriptive analysis was carried out to compare the characteristics of $\mathrm{BB}$ formation between the two groups. Mean and standard deviation (SD) for BB floor thickness are reported.

\section{Results}

A total of 20 human donor corneas were treated, 10 in the TL and 10 in the LT group. Tunnel and lamellar incisions were successful in all cases (Figure 1). Overall, type $1 \mathrm{BB}$ formed in $85 \%$ of corneas, $100 \%$ in the TL and $70 \%$ in the LT group, in which a type 2 BB formed (20\%) and perforation of the Descemet-endothelium complex in one cornea was recorded during cannula insertion. Type $1 \mathrm{BB}$ was achieved after one attempt in $90 \%$ of corneas in the TL group and in 57\% in the LT group (Figure 2). As measured by AS-OCT, the mean ( \pm standard deviation $[\mathrm{SD}]$ ) bubble floor thickness was 27.0 (1.43) $\mu \mathrm{m}$ (range, 25-30) for type $1 \mathrm{BB}$ and 17 and $20 \mu \mathrm{m}$ for type $2 \mathrm{BB}$, respectively.

\section{Discussion}

What is usually dome with FSL-assisted DALK is to prepare the donor and the host cornea for anterior keratoplasty. Studies investigating the value of creating a tunnel in the posterior stroma are few, because current FSL software platforms lack specific software that can shape lamellar and tunnel incisions in sequence for the big-bubble technique. ${ }^{16,18,20}$ To our best knowledge, this ex vivo study is the first to determine whether the order in which the two types of FSL incision is performed during FSL-assisted DALK has an effect on outcome. We noted that the Victus SV-3.4 software provides for the formation of a type 1 bubble and that sculpting the intrastromal tunnel before the lamellar cut enhances its formation in up to $100 \%$ of cases.

The sequence of FSL treatment could affect the type of bubble because of laser-induced optical breakdown (LIOB), a phenomenon that occurs when light pulses interact with optically transparent tissue. ${ }^{21}$ Tightly focused, ultrashort light pulses in femtoseconds produce photo disruption of the tissue and create microcavitation bubbles (MCB) in the treated area. ${ }^{22}$ To create MCB effectively, the tissue needs to be transparent. When FSL works in a previously treated area, the ultrashort light pulses encounter a cloudy dielectric that may act as a barrier to the formation of $\mathrm{MCB}$ and compromise the cut. The tunnel and lamellar cuts intersect with each other. However, when the tunnel incision is performed first, the lamellar incision interferes with it at the level of plane 1. 

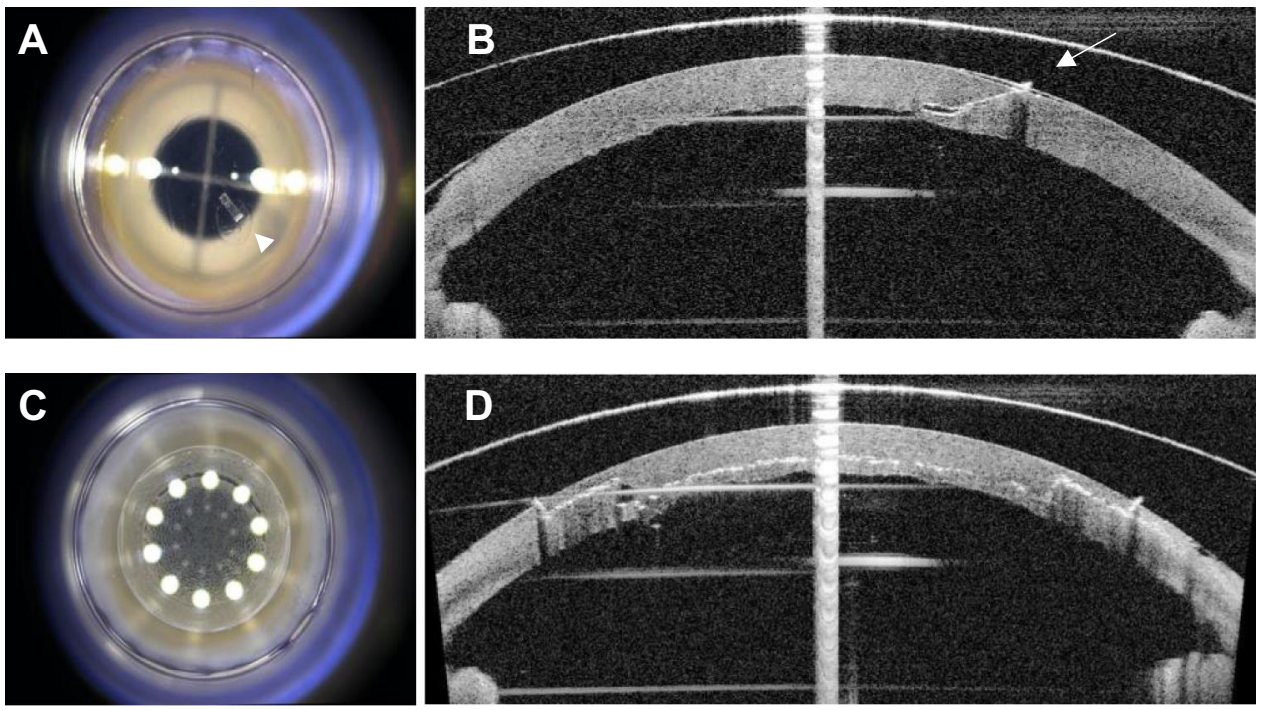

Figure I Surgical microscopy and integrated optical coherence tomography images of tunnel and mushroom FSL incisions. Frontal view of the tunnel incision in the paracentral position at $130^{\circ}$ (A, arrowhead); plane I is angulated while plane 2 lies parallel to the corneal surface (B, arrow); the cut of the internal layers is clear from the frontal view (C); anterior, middle, and posterior view of the lamellar cut following mushroom configuration (D).
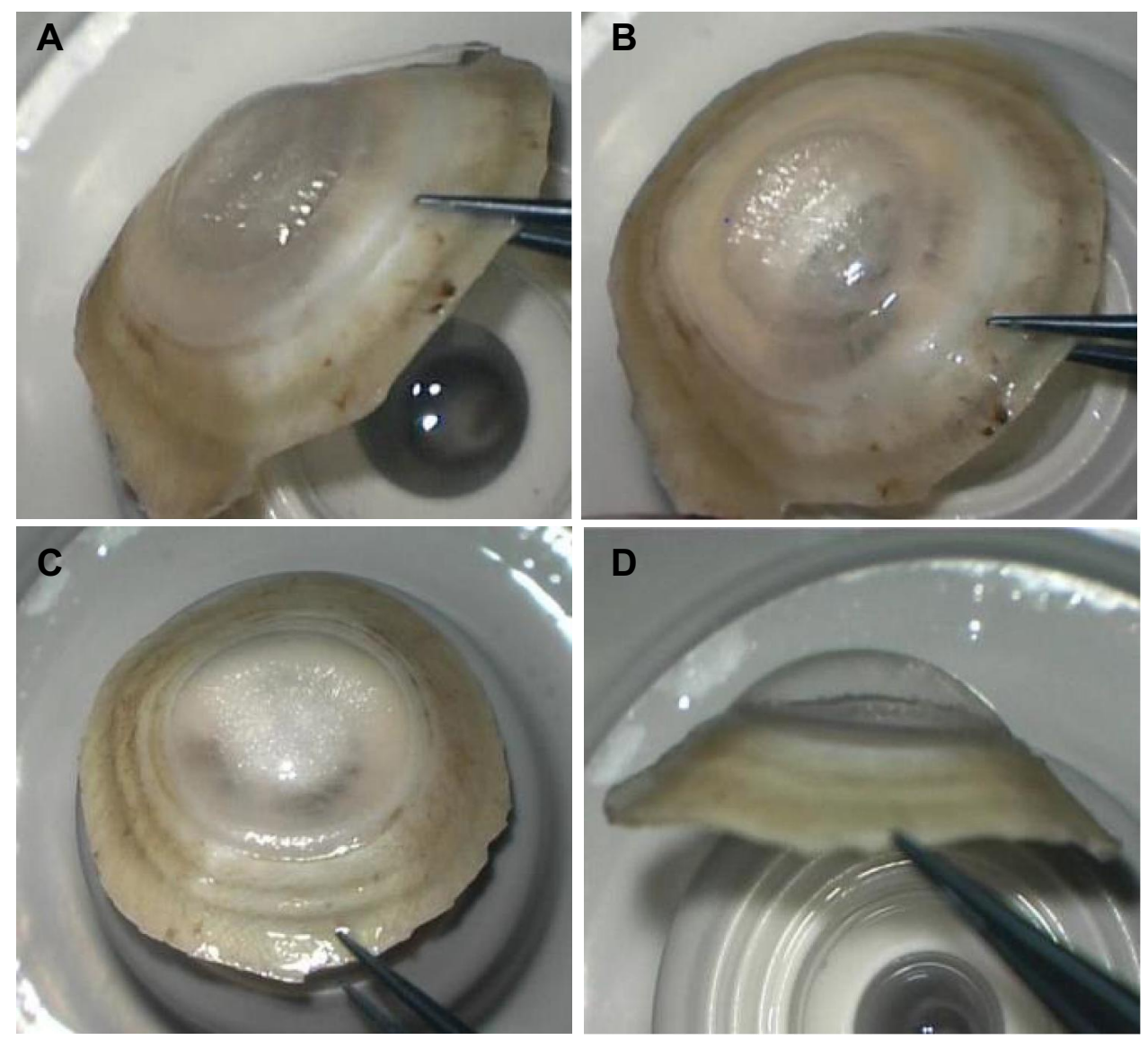

Figure 2 Appearance of type I and type 2 BB after eversion of the cornea. Type I BB, frontal (A) and lateral view (B); type 2 BB, frontal (C) and lateral view (D) showing a smaller diameter type I compared to type 2 bubble.

When the procedure is performed in the reverse order, the tunnel incision is affected by the lamellar cut in plane 1 and along the entire length of plane 2 . This likely results in an incomplete tunnel or increased resistance in plane 2, which can lead to either difficult cannula insertion or its progression due to the need to push the cannula deeper 
without guidance, ensuing in an increase in type 2 BB formation and risk of perforation. ${ }^{23,24}$

In our series, we observed type $2 \mathrm{BB}$ formation in two cases and perforation in one case, all following the LT sequence. Because of the difficulty in advancing the cannula, we were sure we would find the tunnel guide but the quality of the tunnel was actually compromised. While such events do not necessarily compromise the benefit obtained with the laser, they do underline the importance of utmost care during cannula insertion to avoid creating a dissection plane different from the one cut by the laser. In addition, the surgeon will need to carefully advance the cannula tip tangentially beyond the end of the tunnel so that the injected air enters the corneal center and does not flow back and spread into the stromal structure, likely leading to a type $2 \mathrm{BB}$.

When FSL-assisted DALK is performed on patients, the sequence of the FSL cuts is not the only factor to be taken into account to obtain a BB. We recognize that our study findings may not match the data from patients, in which the procedure can be performed for the treatment of corneal disorders that have been shown to influence successful BB formation. ${ }^{9}$

Nonetheless, when shaping the recipient cornea with the Victus SV-3.4 for FSL-assisted DALK, the TL sequence combined with proper insertion of the cannula and air injection may be more effective than the reverse sequence and may avoid an undesirable outcome.

\section{Conclusion}

This study provides ophthalmic surgeons with further evidence for understanding the proper approach toward optimization of the technique in FSL-assisted DALK by pneumo-dissection.

\section{Disclosure}

The author reports no conflicts of interest. The study was supported by Bausch \& Lomb.

\section{References}

1. Ple-Plakon PA, Shtein RM. Trends in corneal transplantation: indications and techniques. Curr Opin Ophthalmol. 2014;25:300-305. doi:10.1097/ICU.0000000000000080

2. Reinhart WJ, Musch DC, Jacobs DS, et al. Deep anterior lamellar keratoplasty as an alternative to penetrating keratoplasty. Ophthalmology. 2011;118:209-218. doi:10.1016/j.ophtha.2010.11.002

3. Anwar M, Teichmann KD. Big-bubble technique to bare Descemet's membrane in anterior lamellar keratoplasty. J Cataract Refract Surg. 2002;28:398-403. doi:10.1016/S0886-3350(01)01181-6

4. Dua HS, Faraj LA, Kenawy MB, et al. Dynamics of big bubble formation in deep anterior lamellar keratoplasty by the big bubble technique: in vitro studies. Acta Ophthalmol. 2018;96:69-76. doi:10.1111/aos. 13460
5. Myerscough J, Friehmann A, Bovone C, Mimouni M, Busin M. Management of type 2 bubble formed during big-bubble deep anterior lamellar keratoplasty. Cornea. 2019;38(6):E20. doi:10.1097/ ICO.0000000000001940

6. Zaki AA, Elalfy MS, Said DG, Dua HS. Deep anterior lamellar keratoplasty - Triple procedure: a useful clinical application of the pre-descemet's layer (Dua's layer). Eye. 2015;29:323-326. doi:10.1038/eye.2014.273

7. Dua HS, Katamish T, Said DG, Faraj LA. Differentiating type 1 from type 2 big bubbles in deep anterior lamellar keratoplasty. Clin Ophthalmol. 2015;9:1155-1157.

8. Altaan SL, Mohammed I, Said DG, Dua HS. Air pressure changes in the creation and bursting of the type- 1 big bubble in deep anterior lamellar keratoplasty: an ex vivo study. Eye. 2018;32:146-151. doi:10.1038/eye.2017.121

9. Scorcia V, Giannaccare G, Lucisano A, et al. Predictors of bubble formation and type obtained with pneumatic dissection during deep anterior lamellar keratoplasty in keratoconus. Am J Ophthalmol. 2020;212:127-133. doi:10.1016/j.ajo.2019.12.012

10. Feizi S, Javadi MA, Daryabari S. Factors influencing big-bubble formation during deep anterior lamellar keratoplasty in keratoconus. Br J Ophthalmol. 2016;100:622-625. doi:10.1136/bjophthalmol-201 5-307111

11. Fogla R. Deep anterior lamellar keratoplasty in the management of keratoconus. Indian J Ophthalmol. 2013;61(8):465-468. doi:10.4103/ 0301-4738.116061

12. Ucgul AY, Yesilirmak N, Yuvarlak A, Aydin B, Ozmen MC, Akata F. Blunt scissors stromal dissection technique compared with big-bubble deep anterior lamellar keratoplasty. Eye Contact Lens. 2019;45(3):195-200. doi:10.1097/ICL.0000000000000557

13. Ibrahim T, Goernert P, Rocha G. Femto-second laser (FSL) techniques and consistency in corneal surgery: experimental study. Can J Ophthalmol. 2018;53(4):324-329. doi:10.1016/j.jcjo.2017.11.005

14. Lu Y, Shi YH, Yang LP, et al. Femtosecond laser-assisted deep anterior lamellar keratoplasty for keratoconus and keratectasia. Int J Ophthalmol. 2014;7(4):638-643. doi:10.3980/j.issn.2222-3959. 2014.04.09

15. Shehadeh-Mashor R, Chan C, Yeung SN, Lichtinger A, Amiran M, Rootman DS. Long-term outcomes of femtosecond laser-assisted mushroom configuration deep anterior lamellar keratoplasty. Cornea. 2013;32(4):390-395. doi:10.1097/ICO.0b013e318254a4e4

16. Buzzonetti L, Petrocelli G, Valente P, et al. The big-bubble full femtosecond laser-assisted technique in deep anterior lamellar keratoplasty. J Refract Surg. 2015;31:830-834. doi:10.3928/1081 597X-20151111-07

17. Guindolet D, Nguyen DT, Bergin C, et al. Double-docking technique for femtosecond laser-assisted deep anterior lamellar keratoplasty. Cornea. 2018;37:123-126. doi:10.1097/ICO.0000000000001442

18. Lucisano A, Giannaccare G, Pellegrini M, et al. Preliminary results of a novel standardized technique of femtosecond laser-assisted deep anterior lamellar keratoplasty for keratoconus. $J$ Ophthalmol. 2020;2020:5496162. doi:10.1155/2020/5496162

19. Fung SS, Aiello F, Maurino V. Outcomes of femtosecond laser-assisted mushroom-configuration keratoplasty in advanced keratoconus. Eye. 2016;30:553-561. doi:10.1038/eye.2015.273

20. Buzzonetti L, Petrocelli G, Valente P. Femtosecond laser and big-bubble deep anterior lamellar keratoplasty: a new chance. J Ophthalmol. 2012;2012:264590. doi:10.1155/2012/264590

21. Janulewicz KA, Rehman ZU, Nguyen VH, et al. Femtosecond optical breakdown as a source of the extreme states of matter. Acta Phys Pol A. 2018;133:292-295. doi:10.12693/APhysPolA.133.292

22. Juhasz T, Kastis GA, Suárez C, Bor Z, Bron WE. Time-resolved observations of shock waves and cavitation bubbles generated by femtosecond laser pulses in corneal tissue and water. Lasers Surg Med. 1996;19:23-31. doi:10.1002/(SICI)1096-9101(1996)19:1<23:: AID-LSM4>3.0.CO;2-S 
23. Scorcia V, Busin M, Lucisano A, et al. Anterior segment optical coherence tomography-guided big-bubble technique. Ophthalmology. 2013;120(3):471-476. doi:10.1016/j.ophtha.2012.08.041
24. Yoo YS, Whang WJ, Kang MJ, et al. Effect of air injection depth on big-bubble formation in lamellar keratoplasty: an ex vivo study. Sci Rep. 2019;9(1):1-8. doi:10.1038/s41598-018-36522-w

\section{Publish your work in this journal}

Clinical Ophthalmology is an international, peer-reviewed journal covering all subspecialties within ophthalmology. Key topics include: Optometry; Visual science; Pharmacology and drug therapy in eye diseases; Basic Sciences; Primary and Secondary eye care; Patient Safety and Quality of Care Improvements. This journal is indexed on PubMed

Submit your manuscript here: https://www.dovepress.com/clinical-ophthalmology-journal
Central and CAS, and is the official journal of The Society of Clinical Ophthalmology (SCO). The manuscript management system is completely online and includes a very quick and fair peer-review system, which is all easy to use. Visit http://www.dovepress.com/ testimonials.php to read real quotes from published authors. 